\title{
El fenómeno WhatsApp en el contexto de la comunicación personal: una aproximación a través de los jóvenes universitarios
}

\section{The WhatsApp phenomenon in the context of personal communication: an approximation through the university youths}

\author{
Juana Rubio-Romero \\ Profesora Doctora de Métodos de investigación en comunicación \\ (Universidad Antonio de Nebrija) \\ Marta Perlado Lamo de Espinosa \\ Decana Facultad de Ciencias de la Comunicación \\ (Universidad Antonio de Nebrija)
}

Fecha de recepción: 24 de mayo de 2015

Fecha de revisión: 7 de junio de 2015

Para citar este artículo: Rubio-Romero, J. y Perlado Lamo de Espinosa, M. (2015): El fenómeno WhatsApp en el contexto de la comunicación personal: una aproximación a través de los jóvenes universitarios, Icono 14, volumen (13), pp. 73-94. doi: 10.7195/ri14.v13i2.818 


\section{Resumen}

La comunicación mediada por el contexto tecnológico y el despliegue digital ha cambiado. El enorme desarrollo de los Smartphone, que ha posibilitado el acceso a internet, ha favorecido el gran desarrollo de la mensajería instantánea hasta el punto que aplicaciones como WhatsApp se han convertido en la principal vía para relacionarse entre los jóvenes, con la ventaja de aportar una comunicación más personal y controlada.

El presente estudio pretende comprender el éxito del fenómeno WhatsApp entre los jóvenes universitarios, explorando las claves de su conquista, así como las actitudes que genera su utilización comparativamente con otros sistemas de comunicación virtual habituales. Para ello, además de revisar los informes y estudios divulgados acerca de esta cuestión, este trabajo se apoya en los resultados del Observatorio cualitativo Jóvenes y Comunicación de la Universidad Nebrija y de una investigación ad-hoc basada en entrevistas y en dinámicas de grupo con jóvenes universitarios.

\section{Palabras clave}

Comunicación online - TIC - Internet - Móvil - Jóvenes - Redes Sociales

\section{Abstract}

This paper pretends to understand the success of the WhatsApp phenomenon among the university youths, exploring the keys of its conquest, and also the attitudes provoked by its use when compared with other commonly used virtual communication systems. In order to do that, apart from carrying out a review of reports and studies on this issue, this study is based on the results obtained from the qualitative Observatory Youth and Communication at Nebrija University and on a research ad hoc based on interviews and group dynamics with university youths.

\section{Key Words}

Online communication - ICT - Internet - Mobile - Youth - Social networks 


\section{Introducción}

Según la $17^{a}$ Encuesta AIMC a Usuarios de Internet, Navegantes en la Red, en 2014 "el teléfono móvil sigue siendo el dispositivo que más gente utiliza para conectarse a internet: el 91,8\% de los encuestados lo hace; y con más frecuencia, durante más tiempo y para más usos que en 2013", se cita en uno de los titulares de la nota de prensa que AIMC facilita con el resumen de resultados de la encuesta. El mismo estudio señala también a la mensajería instantánea entre los servicios más utilizados en el móvil ( $82,9 \%$, con un incremento de 4,7 puntos respecto a 2013); un resultado fruto del liderazgo del software WhatsApp, que ya es utilizado por el $89,4 \%$ de la población internauta.

Asimismo, la VI Ola del estudio sobre Mobile Marketing de la IAB-The Coctail Analysis (2014) señala que WhatsApp incrementa su dominio en mensajería en 2013 en dos puntos respeto al año anterior y figura en primer lugar entre las Apps mencionadas en smartphone (72\%); seguida de Facebook (50\%) y Twitter (26\%).

Por su parte, el Observatorio de Redes Sociales, elaborado por Coktail Analysis y la agencia Arena, en su VI Oleada (2014) sitúa esta aplicación como referencia en cuanto a conversaciones directas, rápidas e inmediatas entre usuarios. WhatsApp supera a Facebook en volumen de internautas con cuenta activa (87\% frente a $83 \%$ ) y consigue ser líder del sector en penetración, acaparando la comunicación privada y de mayor implicación emocional.

Otros datos del mercado confirman lo que representa el fenómeno WhatsApp en España. Según el documento de trabajo sobre servicios Over the Top (OTT) en España, publicado en diciembre de 2014 por la Comisión Nacional de los Mercados y de la Competencia (CNMC), España es el país europeo en el que los usuarios de telefonía móvil usan más los servicios de mensajería instantánea independientes, como WhatsApp, Line u otros similares. De acuerdo con este documento, un 51,5\% de los usuarios de móvil los emplean diariamente y el $83 \%$ de los poseedores de teléfonos inteligentes (smartphone) lo utilizan al menos una vez por semana, muy por encima de otros países como Holanda (67\%), Alemania (43\%), Portugal (34\%), Reino Unido $(30 \%)$, Bélgica $(20 \%)$ y Francia (15\%). Según el mismo informe, este servicio ha

DOI: ri14.v13i2.818| ISSN: 1697-8293 | Año 2015 Volumen 13 № 2 | ICONO14 
supuesto un enorme descenso de los consumos tradicionales de SMS, que han caído un $61 \%$ desde 2011. Entre los que utilizan WhatsApp u otras aplicaciones similares, un $63,3 \%$ no envían SMS, y entre los que sí continúan utilizándolos, el grupo mayoritario es el de los individuos que han reducido su consumo a más de la mitad. Este mismo estudio se hace eco de la brecha generacional en el uso de internet en el móvil, resaltando que son los jóvenes los que hacen un uso más intenso de Internet móvil y los usuarios de edades comprendidas entre los 16 a 34 años los que consiguen mayores tasas de penetración "aproximadamente siete de cada diez usuarios de estas generaciones dispone de Internet sobre el móvil, cuando la tasa en el conjunto de usuarios es del 59,6\%" (CNMC, 2014, p. 11). Según el mismo estudio también son los jóvenes, entre los 16 y los 24 años, los que más usan el servicio de mensajería instantánea $\left(77^{\prime} 3 \%\right)$, frente al conjunto de usuarios $(59,4 \%)$.

Por su parte, Santiago y González (2015) señalan cómo en la actualidad las principales vías de acceso a comentarios sobre programas televisivos en internet son las redes sociales $(66,5 \%)$ y WhatsApp $(42,6 \%)$.

De igual forma, la prensa se hace habitualmente eco de estas tendencias en la comunicación. $A B C(26 / 2 / 2015)$ se ocupa del triunfo de esta aplicación acentuando la comparativa con otros países; "la penetración de WhatsApp en España alcanza un 70\% de usuarios, lo que la sitúa en el cuarto país del mundo por detrás de Sudáfrica (78\%), Singapur (72\%) y Hong Kong (71\%)". Expansión (5/12/2012) se dedica igualmente al fenómeno emergente de WhatsApp con el siguiente titular: “WhatsaApp tumbó a los SMS ¿alguien podrá con WhatsApp?" También es un asunto comentado por los propios lectores que en "Cartas al Director" (El País, 1/3/2015) escriben "WhatsApp hasta en la sopa", dando cuenta de que no sólo el gusto por la charla influye en el éxito de WhatsApp, sino también el alto precio de los tradicionales SMS, un comentario que se cierra con una pregunta: “¿hasta dónde llegará este fenómeno social?”.

Y no sólo a la prensa le interesa este fenómeno, también los jóvenes cineastas tratan esta tendencia. Ejemplos de ello son los cortometrajes Doble check de Paco Caballero (2012) y yo tb tq de Dani Montes (2013), donde se aborda idéntica temática: la incomunicación entre parejas por culpa del WhatsApp; dos trabajos que se convirtieron en su momento en auténticos fenómenos virales.

ICONO14 | Año 2015 Volumen 13 N² 2 | ISSN: 1697-8293 | DOI: ri14.v13i2.818 
Desde una perspectiva cualitativa, los estudios consultados también confirman las cifras referidas y ratifican que WhatsApp se ha convertido en el sistema principal de comunicación instantánea entre los jóvenes.

El informe de la FAD-Injuve (2006) destacaba ya el escaso uso de las herramientas conversacionales más colectivas y anónimas frente a las más personalizadas; una tendencia que confirma las cifras de la AIMC y que el fenómeno WhatsApp pone en evidencia.

En el informe de Telefónica "Los jóvenes en la era de hiperconectividad: tendencias, claves y miradas" se refieren a esta aplicación en los siguientes términos:

El símbolo, el icono más representativo de todo lo que es y significa el smartphone. Una buena música se puede escuchar a través de otro medio, pero esa comunicación inmediata y al alcance de la mano las veinticuatro horas del día, no. Eso lo proporciona el WhatsApp. Es el verdadero valor comunicativo añadido, el plus que se suma al "todo en uno" que concita el Smartphone (2013, p. 95).

Este icono moderno "ha pasado de ser considerado un medio para comunicarse a un fin en sí mismo, lugar en el que tienen lugar las relaciones y escenario en el que también ocurren cosas", se especifica en el Informe de la Fundación Centro Reina Sofía (CRS)-Fundación Ayuda a la Drogadicción FAD (2014, p. 128).

Igualmente desde el Observatorio Nebrija Jóvenes y Comunicación ${ }^{1}$, iniciado en el curso 2008-09, se ha sido testigo directo del ascenso del móvil como acceso a internet y de la aparición del fenómeno WhatsApp. En esta investigación, la aplicación WhatsApp se nombra en los grupos de discusión realizados en $2011^{2}$. Por primera vez, ese año se escucha a los jóvenes universitarios consultados conversar acerca de un sistema de mensajería gratuito a través del móvil, aludiendo a su gran ventaja frente a los mensajes SMS, que hasta el momento eran habituales. No obstante, tanto su uso como el discurso sobre el sistema es exiguo todavía por aquel entonces, y sólo es mencionado por un joven entre los asistentes al grupo de discusión, con el siguiente comentario: “... ahora está el WhatsApp, el chat de la BlackBerry... En mi caso dejo más de lado el Tuenti y el Facebook y eso, y me comunico más por los móviles con estas cosas que son ahora gratuitas". 
Será un año después, en 2012, cuando este sistema de comunicación empieza a adoptarse entre los jóvenes universitarios y ya hablan de WhatsApp con total propiedad, destacando las ventajas de la comunicación móvil e instantánea frente a la que proporcionan las redes sociales. De cualquier modo, es en 2013 cuando los jóvenes universitarios participantes en la investigación destacan esta aplicación para relacionarse con su ambiente cercano por encima de otras formas de comunicación.

Estos mismos resultados se confirman en el hecho de que ninguno de los informes consultados referidos a jóvenes y uso de tecnología, con fecha anterior al 2013, se hacen eco de la aplicación WhatsApp entre las herramientas de comunicación más usadas por los jóvenes.

Tampoco se han encontrado artículos en revistas científicas españolas que se hayan ocupado de este tema en concreto, razón por la que este artículo puede resultar una aportación interesante a esta cuestión, dentro de los consumos tecnológicos de los jóvenes universitarios.

En la investigación que se presenta en este artículo, se parte de unos interrogantes básicos: ¿qué hace que los jóvenes estén todo el tiempo pendientes del WhatsApp?; ¿cuáles son las claves de éxito de esta aplicación entre los jóvenes? Dos interrogantes que conducen a plantear los siguientes objetivos de investigación:

- Indagar acerca de las formas de comunicación más habituales entre los jóvenes universitarios actualmente y sobre las actitudes que se generan hacia la comunicación mediada por la tecnología.

- Explorar la evolución que han experimentado las distintas formas de comunicación conversacional y averiguar las diferencias y semejanzas entre ellas.

- Profundizar en los usos y actitudes hacia la aplicación WhatsApp en concreto. 


\section{Material y métodos}

Para la elaboración de esta investigación se han tenido en cuenta fuentes tanto secundarias como primarias. Las fuentes secundarias nos han ayudado a contextualizar el fenómeno estudiado; las primarias, nos sirven para explorar ad hoc los objetivos planteados.

Las fuentes secundarias han sido fundamentalmente informes técnicos y estudios oficiales realizados por diversas instituciones sobre los jóvenes y las TIC. Entre los estudios consultados destacamos los realizados por la Fundación Telefónica en 2012, Jóvenes, culturas urbanas y redes digitales y en 2013 Los jóvenes en la era de la hiperconectividad: tendencias, claves y miradas, el publicado por la Injuve- FAD, Jóvenes y cultura Messenger en 2006 y por el Centro Reina Sofía (CRS) -Fundación Ayuda Drogadicción (FAD) Jóvenes y comunicación. La impronta de lo virtual en 2014.

Las fuentes primarias han sido de tipo cualitativo; esta metodología nos permite la expresión espontánea de posturas y actitudes de los participantes, además del intercambio dialéctico y la reconstrucción discursiva sobre la realidad social que investigamos. Se ha contado, por un lado, con el trabajo de campo realizado para

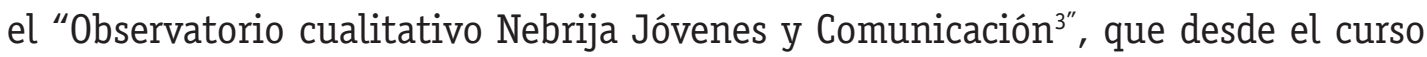
2008-2009 viene realizando dos dinámicas de grupo por curso sobre las relaciones de los jóvenes con las tecnologías de la comunicación. Por otro, se ha realizado un estudio cualitativo ad hoc, centrado en el tema concreto del uso de WhatsApp por parte de los jóvenes universitarios. Para esta investigación ad hoc se han utilizado las dos técnicas básicas de la metodología estructural o cualitativa: grupos de discusión y entrevistas personales.

La técnica del grupo de discusión fue elegida por su gran capacidad de configuración de marcos discursivos amplios, al mismo tiempo que dialécticamente estructurados. Esta técnica ha servido para explorar la influencia de las representaciones sociales en las opiniones espontáneas de los jóvenes, pues, como señalan Canales y Peinado (1995, p. 295), "permite la re-construcción del sentido social en el seno de una situación -grupal- discursiva". Para la conformación de los grupos se

DOI: ri14.v13i2.818 | ISSN: 1697-8293 | Año 2015 Volumen 13 N² 2 | ICONO14 
tomaron en consideración diversas variables: sexo, edad (18-23 años), y el tipo de universidad (pública y/o privada) en la que cursaban los jóvenes sus titulaciones; también se tuvo en cuenta que entre los asistentes a los grupos estuvieran representadas distintas titulaciones y cursos. Se han realizado un total de tres grupos de discusión: dos con alumnos de la Universidad Nebrija y uno con estudiantes de universidades públicas. Los grupos de discusión fueron mixtos (50\% hombres y $50 \%$ mujeres) y estuvieron constituidos por 8-9 participantes cada uno.

Por su parte, las entrevistas personales han resultado más útiles a la hora de indagar sobre aquellos aspectos más pragmáticos, racionales, puesto que es una técnica que, como indica Alonso, L.E. (1995, p.226), permite obtener información de "cómo los sujetos diversos actúan y reconstruyen el sistema de representaciones sociales en sus prácticas individuales". De acuerdo con Herrera y Lizcano (2012, p 29), la entrevista es más productiva que el grupo de discusión "para el estudio de casos típicos o extremos, ciertos individuos que encarnan, con toda su riqueza, el modelo ideal de una determinada actitud, mucho menos cristalizada en la 'media' del colectivo de referencia"; en este caso concreto queríamos contrastar la actitud de los jóvenes respecto a las nuevas tecnologías, diferenciando entre integrados y críticos. De este modo, se han realizado un total de 4 entrevistas en profundidad; dos a jóvenes más integrados y las otras dos a jóvenes más críticos, 50\% de hombres y $50 \%$ mujeres.

Así pues el total del trabajo de campo realizado para el estudio ad hoc han sido 3 grupos de discusión y 4 entrevistas en profundidad. Se puede llegar a pensar que se trata de una muestra muy escasa y no representativa, por lo que nos parece necesario aclarar que el concepto de representatividad en investigación cualitativa no es el mismo que el que se aplica en investigación cuantitativa. La muestra cualitativa no busca una representatividad estadística sino estructural; puesto que lo que pretende comprender son los distintos sentidos sociales (imaginario social) de los grupos de referencia y las relaciones de la población con el fenómeno investigado, y no la extrapolación de los datos al universo, como en el caso de la muestra cuantitativa. Para comprobar la representatividad de la muestra cualitativa tenemos que considerar la saturación del discurso, es decir, cuando deja de producirse información nueva relevante sobre el objeto de estudio. En este sentido, y sobre el

ICONO14 | Año 2015 Volumen $13 N^{\circ} 2$ | ISSN: 1697-8293 | DOI: ri14.v13i2.818 
objeto de la presente investigación, se ha encontrado un discurso muy redundante y uniforme, con pocas diferencias significativas entre las distintas variables que se han tenido en cuenta en el diseño de la muestra, lo que llevó a modificar el diseño inicial previsto (4 grupos de discusión y 6 entrevistas en profundidad) cuando, una vez consideradas todas las variables, advertimos que ya no se aportaba información adicional relevante al análisis.

\section{Resultados}

\subsection{Lo presencial y lo virtual en el contexto de la comunicación personal en los jóvenes: entre la tensión y la complementariedad}

De forma generalizada, los jóvenes destacan las ventajas y comodidades que suponen el uso de las herramientas tecnológicas para la comunicación personal, pero también perciben las pérdidas que conlleva este tipo de comunicación frente a la presencial, cara a cara, que sigue siendo el modelo de las relaciones "auténticas", aquellas capaces de transmitir emociones. La comunicación mediada por la tecnología es más "impersonal" y susceptible de malentendidos, opinan.

Los jóvenes justifican que, ya sea por comodidad, por falta de tiempo o por problemas de timidez, se comunican más por WhatsApp que cara a cara, si bien cuando se trata de asuntos importantes y personales, la comunicación presencial es indispensable, lo que no impide que en los grupos de discusión se narren multitud de experiencias de discusiones de parejas por WhatsApp, tal y como se recoge a continuación:

“...entonces, tú devuelves otra cosa que también es mal interpretada y entras en un círculo de una discusión sin sentido que realmente habría sido una idiotez si estuvieseis los dos juntos. ¿Sabes lo que te digo? 0 si coges el teléfono y llamas..."

Respecto a la utilización de tecnología para la comunicación surgen sentimientos encontrados; por un lado, ésta mejora las relaciones al facilitar el acercamiento conversacional, mucho más evidente cuando se asocia a algunos problemas de los usuarios, para los cuales sería un facilitador ${ }^{4}$ que ayuda a superar la timidez. Así lo relata uno de los participantes en la investigación:

DOI: ri14.v13i2.818 | ISSN: 1697-8293 | Año 2015 Volumen 13 N² | ICONO14 
“... lo que tiene también es que a la gente que le cuesta comunicarse cara a cara, lo digo por experiencia, le va mejor. Porque yo conozco gente que en persona es que ni está, y luego por WhatsApp son personas totalmente diferentes. Igual luego se sienten más tranquilos, igual por Facebook, delante del ordenador... Depende de lo tímido que sea... ¿sabes? Les cuesta menos. También es un medio de relación".

Los jóvenes asocian la conversación virtual a escribir mensajes (excepto por Skype) pero la comunicación escrita carece de los elementos reales, como gestos o movimientos que acompañan a la comunicación oral y que, señalan, son los principales encargados de transmitir emociones. Para tratar de paliar estas limitaciones se utilizan otros recursos, como emoticones, signos de puntuación o elementos multimedia, en un esfuerzo por complementar el sentido de lo que transmiten. Sin embargo, resulta hasta cierto punto paradójico cómo, teniendo en cuenta que los smartphones, que permiten la posibilidad de usar mensajes de voz en lugar de escritos, esta función apenas aparezca en los discursos de los jóvenes.

Este target considera que la comunicación mediada por la tecnología les aleja de las relaciones más humanas, pero justifican y comprenden su necesidad por el tipo de vida actual. Otra cuestión es que se abuse de esta tecnología facilitadora hasta el punto de llegar a suplir las relaciones presenciales, tal y como se refieren al respecto alguno de los participantes en la investigación:

“...el hecho de que la comunicación ahora sea, entre comillas, gratuita, no significa que nos comuniquemos más..., no porque ahora tengamos WhatsApp nos comunicamos más. Creo que cada vez se hacen menos reuniones de amistad; las cosas importantes ya no se tratan en persona, ya que todo es a golpe de tecla".

“...yo hablo con mi compañera de piso desde la habitación de al lado”.

Para los jóvenes, los dos entornos, el virtual (online) y el real o presencial (offline) son intercambiables y ambos forman parte de sus vidas cotidianas; consideran que lo real y lo virtual no se oponen sino que están interconectados. “...Yo pienso como él, conocer a una persona digitalmente me crea más simpatía, porque ya la he mirado con otros ojos. 0 al revés, más antipatía", manifiestan. 
Y ya sea porque se solapen o porque lo online se utilice como mediador del entorno offline, lo cierto es que internet es el primer paso en el camino de cualquier tipo de relación y un medio para tantear la situación, tal y como afirma uno de participantes en el estudio: "Utilizas redes sociales o WhatsApp como unión para luego decir quedamos en tal sitio".

Incluso se puede llegar a construir en paralelo a la persona real, otro yo virtual, más íntimo y personal, y actuar desde él en la comunicación online. Parece que es posible incluso para algunos la construcción de un clima de mayor intimidad, pues al no sentirse coartados por la presencia física del otro, se expresan con absoluta libertad. Así lo relatan en sus exposiciones:

"Yo conozco una chica que me llama muchísimo la atención, hablas con ella y es súper tímida, nunca dice nada y en Twitter es TuitStar ¿sabes? Y dices, ipuf!, yo qué sé..., se te da mejor el mundo virtual..."

“Pero eso le pasa a mucha gente. En Twitter es diferente, porque en Twitter pones frases... WhatsApp es más comunicación"

Para la comunicación virtual, WhatsApp y las redes sociales son los sistemas más habituales para relacionarse con los amigos; también se menciona Skype para una interacción más íntima y en un entorno más reposado. De estos tres, sin duda, Whats$A p p$ es el más común y normal porque es el más manejable para lo conversacional.

“Es más cómodo el WhatsApp porque no tienes por qué responder en seguida. 0 sea, ponte que estoy estudiando y me llega un mensaje al WhatsApp, pues puedo dejarlo ahí y ya contestaré después. Sin embargo, si me llaman por Skype, ya tengo que parar y contestar".

"Yo creo que muchas relaciones, de amistad y familiares, sobreviven gracias al Skype, por ejemplo; por poner otro que no sea WhatsApp. Poder verte gratis..."

Por el contrario a estos sistemas virtuales, la llamada telefónica está totalmente en desuso y ha sido sustituida por WhatsApp (igual que ha pasado con los SMS en otros 
tiempos tan utilizados). Tal es así que para los jóvenes, el número de teléfono hoy es más un contacto a través de WhatsApp que una conversación telefónica, algo que parece haber quedado fuera las reglas de comportamiento habitual de la juventud.

“La gente ya no te dice: dame el móvil, si no que te piden el WhatsApp o tu Facebook. Ya lo que es tu número, como número de teléfono antiguamente, ya no te lo piden".

Pareciera que a través del WhatsApp les resultara más fácil relacionarse porque lo real únicamente está presente como simulacro, conversan y se expresan como si estuvieran presentes, pero no lo están, falta toda la calidez y emocionalidad que tiene la comunicación presencial, pero también es más fácil expresar los sentimientos preservados tras una pantalla.

Otro aspecto que los jóvenes destacan en relación al uso de WhatsApp es una cierta compulsión a estar permanentemente comunicándose lo que motiva una cierta frivolidad conversacional, tal y como lo describe uno de los jóvenes:

“Antes se pensaba cuándo y a quién llamar, a qué hora, el motivo de la llamada. Sin embargo ahora da igual la hora o el motivo para escribirte por WhatsApp, por ejemplo, ¿Hola qué haces?"

“Antes si tenías que enviar un mensaje como que te lo pensabas más, ya no sólo por el dinero, sino que en plan..., si me gusta un chico, pues voy a enviar un mensaje que esté perfecto y tal; ahora tienes WhatsApp, te da igual hablarle porque como es gratis y todo el mundo lo hace, te da igual decir sólo un 'qué tal', no tienes por qué llenar el mensaje ni nada".

Estos comportamientos compulsivos no hacen sino poner de manifiesto la emergencia de la función fática (Alonso y Conde, 2002), aquella que sirve para facilitar el contacto físico o psicológico entre los sujetos. Así lo evidencia uno de los jóvenes en su discurso:

“Yo, por ejemplo, que no soy de Madrid, lo que sí he notado es que tengo mi grupo, como tiene casi todo el mundo, mi grupo de amigos del pueblo, en el Whats-

ICONO14 | Año 2015 Volumen $13 N^{\circ} 2$ | ISSN: 1697-8293 | DOI: ri14.v13i2.818 
App, entonces pues antes echaba de menos a mis amigos y ahora no. 0 sea, sí echo de menos, a lo mejor, abrazar a mis amigos, pero es que no, es que ya no les echo de menos, porque sé perfectamente lo que hacen en cada momento, me mandan fotos de donde están, de lo que hacen, o sea que ya es más, a veces, echarlos de más..."

Y es que, ciertamente, como manifiestan Alonso y Conde (2002), dicha función de contacto, si bien siempre ha estado presente, ha pasado a ocupar en la actualidad un lugar preeminente, y ello porque, debido a la ruptura de "pacto simbólico" y del liberalismo exacerbado que rige el modelo social actual, se derivan determinadas consecuencias como el aislamiento, la agudización del individualismo, la ausencia de relaciones auténticas, falta de referentes externos, etcétera, que reclaman la necesidad de contacto ${ }^{5}$ con los otros como forma de superar y adaptarse a esta nueva realidad, por lo que WhatsApp, al igual que el resto de las TIC, se inscriben entre lo que estos autores denominan "bienes fáticos". El siguiente verbatim de uno de los jóvenes lo enuncia muy claramente:

“...Para mí, en mi vida cotidiana, WhatsApp es súper importante. Yo me levanto y lo primero que hago es enviar WhatsApps a mis amigas y al irme a dormir también, por la noche, o si me levanto por la mañana, miro la hora, y el WhatsApp también lo miro, a ver qué se cuece..."

\subsection{WhatsApp: diferencias y semejanzas con otros sistemas de interacción personal entre los jóvenes}

WhatsApp no es sólo un medio de comunicación interpersonal entre los jóvenes sino que hablar a través de esta aplicación se ha convertido en una forma de comunicarse; un espacio donde se tienen experiencias.

Los jóvenes están pendientes de este espacio las 24 horas del día, como así lo manifiestan:

“El WhatsApp es básico y clave y tienes que estar las 24 horas pendiente de que pueda haber cualquier tipo de comunicación con esa persona".

DOI: ri14.v13i2.818 | ISSN: 1697-8293 | Año 2015 Volumen 13 N² | ICONO14 
La actitud positiva de los jóvenes universitarios participantes en la investigación hacia la comunicación por WhatsApp hay que inscribirla en el contexto de "progreso" que supone esta aplicación frente a otras formas de comunicación, tecnológicas y no tecnológicas. Si lo comparamos con la comunicación cara a cara, la conversación por WhatsApp, más allá de diferencias señaladas, guarda similitudes, pues se expresan de modo semejante a como lo harían de forma presencial (cara a cara).

“Es que muchas veces es hablar por hablar: “¿qué haces? "Viendo la tele ¿Y tú? Yo también"... Como hablarías estando con esa persona".

Si hacemos la comparativa de WhatsApp con las conversaciones que mantienen a través de los chats en las redes sociales, las similitudes son más que evidentes, hasta el punto que los jóvenes se refieren indistintamente a estos dos sistemas de comunicación $\mathrm{n}^{6}$; aunque se tienen claras las diferencias conceptuales, WhatsApp no es una red social sino un servicio de mensajería instantánea, pero la consideración, en cuanto a uso comunicativo, es la misma:

“Yo, por ejemplo, soy un consumidor mucho más tradicional. Prácticamente redes sociales no uso, o sea las tengo creadas pero no les doy uso... Bueno a la única que le doy uso es al WhatsApp, que yo por lo menos lo considero red social".

“WhatsApp, realmente es un chat móvil".

Otra semejanza entre WhatsApp y las redes sociales es que ambas permiten compartir, participar, tal y como se recoge uno de nuestros participantes:

“...compartes vídeos, información, artículos, ya no es únicamente un intercambio de persona a persona, de mi información de mi cabeza a la tuya... son cosas que hacen que tengas un recuerdo ¿sabes?, algo así como que te apetece compartir: fotos, vídeos, todo..."

Frente a Facebook, los jóvenes manifiestan varias diferencias; en primer lugar, WhatsApp se considera para una interacción más personal $\mathrm{y}$, en este sentido, se percibe más "controlable" y con menor exposición de datos personales:

ICONO14 | Año 2015 Volumen $13 N^{\circ} 2$ | ISSN: 1697-8293 | DOI: ri14.v13i2.818 
“Es individual, tú con esa persona, se pueden enviar, en cambio en Facebook eres tú, hacia el mundo o tus amigos".

“Es una forma de comunicación rápida, no expones datos personales, salvo el número de teléfono...".

Es además un medio de comunicación más directo, sencillo y en movilidad:

“...Yo uso WhatsApp, pero, por ejemplo, para hablar con la gente no utilizo Facebook. Me acuerdo que cuando era pequeño utilizaba Tuenti para hablar con todo el mundo y ahora que ha entrado WhatsApp, entras, hablas, guardas el móvil y luego lo vuelves a mirar cuando te dé la gana, no tienes la necesidad de tener que encender el ordenador. En el móvil está Facebook, está... pero es mucho más coñazo que el WhatsApp, que es mucho más directo".

Asimismo, WhatsApp se utiliza para comunicarse con un tipo de gente más heterogéneo, de manera que no se limita a conversar con los amigos, como es el caso de Facebook. Sea como fuere, lo cierto es que por los jóvenes; Facebook es más para contar historias y WhatsApp es para hablar y comunicarse.

Además de la comunicación cara a cara y de las redes sociales, WhatsApp también está relacionada con otras tecnologías del pasado reciente, de las que los jóvenes piensan que es deudora. Todos recuerdan lo que supuso Messenger, que revolucionó las formas de comunicación y fue la antesala de lo que luego llegaría, las redes sociales; y también recuerdan cómo este sistema innovador en su momento fue abandonándose cuando Tuenti lanzó su chat.

El Messenger significó para los jóvenes su introducción en una nueva forma de comunicación y de relación, de gestionar sus redes de amigos, generando toda una "cultura Messenger" (Gordo y Megías, 2006) que permanece viva en su recuerdo y con la que se suscitan vínculos afectivos muy positivos.

Con la integración de lo conversacional en las redes sociales, el Messenger de pronto perdió notoriedad entre los jóvenes frente a la comodidad del chat de la red 
social, a través del cual podían conversar con los amigos sin necesidad de cambiar de aplicación. Se daba por supuesto que los amigos estaban en Tuenti, la red social de los jóvenes por antonomasia hasta 2011-12; después ha quedado como la red social de los adolescentes.

Otro antecedente del WhatsApp son los SMS por móvil, una forma de comunicación de enorme éxito entre los jóvenes hasta que apareció WhatsApp y empezó su declive. Frente a los SMS, WhatsApp ofrece muchas ventajas y supone un gran avance por lo que ha conseguido "borrar del mapa" a los SMS, a los que se refieren utilizando un discurso peyorativo y agresivo, muy diferente al que emplean para referirse al Messenger:

“WhatsApp lo que hizo fue borrar del mapa los SMS... Y lo hizo de una forma muy buena porque empezó siendo gratis y lo acabaron cobrando a un euro, pero un euro nos parece una miseria porque cada SMS nos salía a 20 céntimos. Yo creo que sustituye a los SMS, pero complementa a cualquier red social".

Por otro lado, así como en el pasado se daba por hecho que los amigos estaban en la red social del momento, Tuenti, hoy también se da por supuesto que todos los contactos personales tienen WhatsApp.

Recientemente han aparecido otras marcas de mensajería instantánea, como Line, que los jóvenes conocen bien y han probado, pues están muy al tanto de todas las novedades tecnológicas, pero, aunque Line aporta algunas mejoras, no ha supuesto una gran innovación y todavía goza de poca implantación.

Un último aspecto del WhatsApp, que provoca una enorme ambivalencia entre los jóvenes, es el que se refiere a la posibilidad que ofrece la aplicación de controlar al otro; saber la hora de conexión o si ya ha recibido y leído el mensaje (con la marca del doble check); una cuestión que parece ser fuente de conflictos, como así lo manifiestan:

"Yo soy relativamente nueva en el mundo del WhatsApp y me he planteado seriamente desactivarlo o cambiar al móvil antiguo que tenía antes. Porque me ha

ICONO14 | Año 2015 Volumen $13 N^{\circ} 2$ | ISSN: 1697-8293 | DOI: ri14.v13i2.818 
creado una serie de conflictos que antes no había, del tipo "te has conectado, lo has leído y no me has contestado desde hace no sé cuántas horas..."

“... se ve cuando te has conectado por última vez y todo eso. Y entonces ya estás ahí nerviosa porque no te han contestado o lo que sea, y no sabes por qué, entonces te enfadas, y te deprimes".

En este sentido, es cierto que los jóvenes saben que pueden configurar esta función simplemente eliminándola, pero si lo hacen, entienden que, por un lado, esto mismo puede ser fuente de conflicto $\mathrm{y}$, por otro, eliminarlo implica que ellos mismos no pueden acceder a este tipo de información sobre los otros, algo a lo que no parecen estar dispuestos a renunciar.

Más allá de estos "inconvenientes", lo que deja claro la investigación es que WhatsApp se ha convertido en la herramienta de comunicación por excelencia para los jóvenes y no solo para mantener conversaciones sino también para intercambiar información, lo que la acerca mucho a una red social, con la ventaja de que es más personal, más rápida y sencilla de usar en movilidad.

\section{Discusión}

Este trabajo arrancaba del planteamiento de estudio acerca del éxito del fenómeno WhatsApp entre los jóvenes universitarios, sondeando las posibles claves de conquista entre este público objetivo. La investigación establecía asimismo tres objetivos concretos cuyas conclusiones se sintetizan a continuación.

En primer lugar, el estudio pretendía averiguar cuáles son las formas de comunicación más habituales entre los jóvenes universitarios y qué actitudes tienen los jóvenes hacia la comunicación mediada por la tecnología. En este sentido, a través de la investigación se confirma cómo los dos entornos de comunicación actual, el virtual y presencial, son igualmente comunes y forman parte habitual de la vida cotidiana de los jóvenes, considerando que entre ellos lo real y lo virtual no se oponen sino que se complementan perfectamente.

DOI: ri14.v13i2.818| ISSN: 1697-8293 | Año 2015 Volumen 13 № 2 | ICONO14 
Entre las formas de comunicación virtuales, las redes y WhatsApp son los sistemas más habituales con las que los jóvenes se relacionan con los amigos, puesto que Skype se utiliza más en un espacio más íntimo que requiere más tiempo y atención. De las tres, WhatsApp es la más utilizada pues es la que mejor se adapta al uso en movilidad; es la forma de comunicación que resulta más cómoda y práctica, hasta el punto que casi ha sustituido la llamada telefónica, que ha quedado desterrada de las formas de comunicación juveniles. En el estudio aparece el hecho paradójico de la falta de utilización de los mensajes de voz en los smartphone, evidenciando cómo el medio habitual de los jóvenes es ahora el teclado y los caracteres, más allá de la voz y de la presencia física.

En relación a las actitudes que manifiestan hacia la comunicación mediada por la tecnología, ésta la conceptúan inicialmente de fría aunque la utilizan habitualmente por su ritmo de vida, más incluso que cara a cara, pues el encuentro presencial está reservado para temas personales de mayor calado y más personales. Evidentemente los jóvenes recalcan cómo la comunicación mediada por la tecnología es más proclive a las tergiversaciones que la personal y en esta línea señalan también los riesgos del uso/abuso de este tipo de comunicación, siendo conscientes de que no se comunican más que antes, sino de otro modo, llegando a reconocer esa cierta banalidad conversacional en la que a menudo incurren.

Por otro lado, a la comunicación virtual la reconocen como facilitadora del acercamiento pero al ser más impersonal es igualmente más demandante de esfuerzo para complementar el sentido de los mensajes.

Un segundo objetivo de la investigación era el de explorar la evolución que han experimentado las distintas formas de comunicación conversacional y examinar las diferencias y semejanzas entre ellas. Respondiendo a este propósito, hay que destacar cómo el adelanto tecnológico que ha supuesto WhatsApp frente a otras formas de comunicación entre los jóvenes es una de sus claves de éxito.

Comparativamente con el Messenger y los SMS del pasado, es un avance por su gratuidad, integración de servicios audiovisuales, posibilidad de comunica-

ICONO14 | Año 2015 Volumen $13 N^{\circ} 2$ | ISSN: 1697-8293 | DOI: ri14.v13i2.818 
ción grupal y universo de usuarios. Ciertamente, los jóvenes recobran en una única aplicación algunas de las funciones que las herramientas del pasado les daban; a través de WhatsApp pueden conversar, compartir e interactuar grupalmente en movilidad, además de ser una aplicación muy fácil de manejar y muy implantada.

En relación a las diferencias y semejanzas entre las formas de comunicación conversacional en el entorno virtual, WhatsApp se acerca a los usos que hacen de las redes sociales en cuanto a la participación y distribución de información, aunque se sepa que no lo es, sino que se trata de una aplicación de mensajería instantánea.

De ella además valoran su uso más individualizado y su cariz heterogéneo, puesto que con WhatsApp el universo se amplía más allá de los jóvenes o los amigos. Asimismo, comparativamente con la conversación cara a cara, en esta aplicación el diálogo llega a ser muy similar al de cualquier conversación entre dos o más personas que físicamente se encuentran, aportando vídeos o imágenes que enriquecen la conversación.

Por último, el estudio se planteaba profundizar en los usos y actitudes hacia la aplicación WhatsApp en concreto. Pues bien, en este sentido los resultados revelan cómo esta aplicación no es sólo una nueva forma de comunicación instantánea sino un espacio de experiencias compartidas, donde se inician, se mantienen y/o se rompen amistades o relaciones. Un medio plagado de ventajas, a pesar de algunos reparos como el uso compulsivo y la exigencia que sienten de estar siempre conectado.

Sin duda, y a pesar de estas objeciones, WhatsApp es la herramienta de comunicación instantánea más valorada y utilizada por los jóvenes en este momento. Su capacidad, facilidad de uso, gratuidad, universalidad, movilidad e innovación parecen ser algunas de sus claves de éxito para situarla en primer lugar en el escenario de las nuevas formas de comunicación actuales. 


\section{Notas}

[1] Este Observatorio, que cada año actualiza el trabajo de campo incorporando un mínimo de dos grupos de discusión con jóvenes universitarios, está coordinado por Juana Rubio-Romero, una de las autoras de este artículo, que imparte la asignatura de "Métodos de investigación en comunicación". Parte de la información que proporciona el Observatorio está incorporada a los resultados de la investigación que se presentan en este trabajo.

[2] WhatsApp, fundada en 2009, se afianza en España unos años después. En 2011 empieza a registrarse un descenso significativo del 33\% (fuentes: CMT y 0ntSi) en el uso de SMS y entre 2012-2013, con la popularización de los smartphones, es cuando se produce la eclosión del fenómeno WhatsApp en nuestro país. El Observatorio citado no lo registra hasta el 2011 y los estudios cualitativos publicados que se han consultado no lo hacen hasta 2013; probablemente porque el trabajo de campo de los estudios publicados es siempre anterior; también puede influir el que el target de estudio se limita a los jóvenes universitarios, grupo early adopters en este tipo de productos, más en el caso de una universidad privada como la Universidad Nebrija.

[3] Dicho Observatorio, cada curso, además de las dinámicas de grupo, suele enriquecerse con el trabajo que realizan los propios estudiantes de $4^{\circ}$ curso de los tres grados de la Facultad de Ciencias de la Comunicación; Comunicación Audiovisual, Periodismo, y Publicidad y RR.PP., en los proyectos que desarrollan para la asignatura de Metodologías de Investigación.

[4] En este estudio de Gordo y Megías (2006, p.151), los autores hablan incluso de uso "terapéuti$\mathrm{co}^{\prime}$, en tanto que sirven para "aliviar la soledad de personas que no pueden salir de casa (por enfermedades, por fobias sociales, o simplemente porque tus padres no te dejan), o que son nuevas en una ciudad y no conocen a nadie".

[5] La necesidad de contacto maternal del bebé expresa bien esta experiencia humana.

[6] Según el V Estudio anual de Redes Sociales, publicado por IAB Spain, en abril de 2014, el 59\% de los encuestados considera que WhatsApp es una red social por la posibilidad de comunicarse con otros, frente al $41 \%$ que no la considera, por ser un chat, sustituto de los SMS o por ser privado sólo para tus contactos.

\section{Referencias}

ABC (26 de febrero de 2015). España, el cuarto país del mundo en el uso del WhatsApp. Recuperado de: http://www.abc.es/tecnologia/moviles-aplicaciones/20150225/ WhatsApp-espana-cuarto-pais-mundo-cuota-mercado-201502241120.html AIMC (2015). Crece el consumo de televisión a través del teléfono móvil y la tablet entre la población internauta. AIMC presenta los resultados de su $17^{a}$ Encuesta a Usuarios de Internet. [Nota de prensa del 12/3/2015]. Recuperada de: http:// www.aimc.es/-navegantes-en-la-red-.html

ICONO14 | Año 2015 Volumen 13 Nº 2 | ISSN: 1697-8293 | DOI: ri14.v13i2.818 
El fenómeno WhatsApp en el contexto de la comunicación personal | 93

AIMC (2015). Navegantes en la Red - Encuesta AIMC a usuarios de Internet $17^{a}$.

Madrid. Recuperado de: http://download.aimc.es/aimc/974_ryRa6/ macro2014ppt.pdf

Alonso, L.E. (1995). "Sujeto y discurso: el lugar de la entrevista abierta en las prácticas de la sociología cualitativa". En Delgado, J.M. y Gutiérrez, J. (Eds.), Métodos y Técnicas cualitativas de investigación en Ciencias Sociales (pp. 225240). Madrid: Síntesis.

Alonso, L.E. y Conde, F. (2002). Gente conectada: la emergencia de la dimensión fática en el modelo de consumo glocal. Política y Sociedad, 39 (1), 27-51.

Caballero, P. (Director) (2012) Doble Check [Cortometraje]. España. Recuperado de: http://www.notodofilmfest.com/ediciones/x/index.php?corto=32360\#/ Home/Ficha/32360/

Canales, M., y Peinado, A. (1995): "Grupos de discusión". En Delgado, J.M. y Gutiérrez, J. (Eds.), Métodos y Técnicas cualitativas de investigación en Ciencias Sociales (pp. 287-316). Madrid: Síntesis.

Comisión Nacional de los Mercados y la Competencia (CNMC). (2014). Caracterización del uso de algunos servicios Over The Top en España. (Documento de Trabajo $\mathrm{n}^{0}$ 4). Recuperado de: http://cnmcblog.es/wp-content/uploads/2015/01/ doc_ott_1_12.pdf.

Comisión Nacional de los Mercados y la Competencia (CNMC). (2014). Informe Económico de las Telecomunicaciones y del Sector Audiovisual 2014. Recuperado de: http://informetelecom.cnmc.es/docs/informe\%20economico\%20sectorial/ informe $\% 20$ telecomunicaciones $\% 20 \mathrm{cnmc} \% 202014$.pdf

Expansión (5 de diciembre de 2012). WhatsApp tumbó a los SMS, ¿Alguien podrá con WhatsApp?. Recuperado de http://www.expansion.com/2012/12/05/ empresas/digitech/1354725006.html

García Canclini, N.; Cruces, F. y Urteaga, M. (Coord.) (2012). Jóvenes, culturas urbanas y redes digitales. Prácticas emergentes en las artes, las editoriales y la música. Madrid: Fundación Telefónica. Recuperado de: http://www.fundaciontelefonica.com/ arte_cultura/publicaciones-listado/pagina-item-publicaciones/?itempubli=182

Gordo López, A. y Megías Quirós, I. (2006). Jóvenes y cultura messenger. Tecnología de la información y la comunicación en la sociedad interactiva. Madrid: INJUVEFAD. Recuperado de: http://www.injuve.es/observatorio/infotecnologia/ jovenes-y-cultura-messenger

DOI: ri14.v13i2.818 | ISSN: 1697-8293 | Año 2015 Volumen 13 N² | ICONO14 
Herrera Racionero, P. y Lizcano Fernández, E. (2012). Apuntes sobre metodología y técnicas cualitativas aplicadas a la investigación socioambiental. Intersticios: Revista Sociológica de Pensamiento Crítico. 6 (1), 25-41

IAB (2014). V Estudio anual de Redes Sociales. Recuperado de: http://www. iabspain.net/wp-content/uploads/downloads/2014/04/V-Estudio-Anual-deRedes-Sociales-versi\%C3\%B3n-reducida.pdf

IAB y The Coktal Analysis (2014). VI Estudio Anual Mobile Marketing. Recuperado de: http://www.iabspain.net/wpcontent/uploads/downloads/2014/09/VI_ Estudio_Anual_Mobile_Marketing_version_abierta1.pdf

Megías Quirós, I. y Rodríguez San Julián, H. (2014). Jóvenes y comunicación. La impronta de lo virtual, Madrid: Centro Reina Sofía-FAD. Recuperado de: http:// ep00.epimg.net/descargables/2014/04/09/93327975ca1922bb61cee54b996a0 01c.pdf.

Montes, D. (Director) (2013) Yo tb tq [Cortometraje]. España. Recuperado en: https://www.youtube.com/watch?v=HsDjCddrz1w

Prior Muñoz, V. (1 de marzo de 2015). WhatsApp hasta en la sopa. [Cartas al Director]. El País. Recuperado de: http://elpais.com/elpais/2015/02/27/ opinion/1425056140_451216.html

Reig, D. y Vilches, L.F. (2013). Los jóvenes en la era de la hiperconectividad: tendencias, claves y miradas. Madrid: Fundación Telefónica. Recuperado de: http://www.fundaciontelefonica.com/arte_cultura/publicaciones-listado/ pagina-item-publicaciones/?itempubli=182.

Santiago, F. y González, I. (febrero, 2015). Nuevos tiempos y nuevos usos del tiempo libre en torno al consumo audiovisual. Ponencia presentada en $31^{\circ}$ Seminario de Televisión AEDEMO. AIMC. Sevilla.

The Coktal Analysis y Arena (2014). Observatorio de Redes Sociales. (VI Ola). Recuperado de: http://tcanalysis.com/blog/posts/the-cocktail-analysis-yarena-publican-la-vi-ola-del-observatorio-de-redes-sociales 